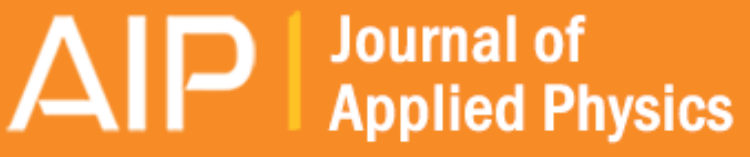

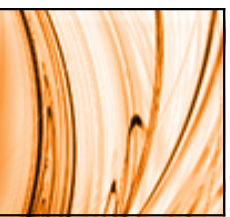

Substrate lattice relaxations, spectral distortions, and nanoparticle inclusions of ion implanted zinc oxide

Y. Wang, B. Ma, W. Zhang, D. Li, Y. Zhao, A. A. Finch, and P. D. Townsend

Citation: Journal of Applied Physics 118, 095703 (2015); doi: 10.1063/1.4929826

View online: http://dx.doi.org/10.1063/1.4929826

View Table of Contents: http://scitation.aip.org/content/aip/journal/jap/118/9?ver=pdfcov

Published by the AIP Publishing

\section{Articles you may be interested in}

Real space pseudopotential calculations for size trends in Ga- and Al-doped zinc oxide nanocrystals with wurtzite and zincblende structures

J. Chem. Phys. 141, 094309 (2014); 10.1063/1.4893478

Ferromagnetic structurally disordered $\mathrm{ZnO}$ implanted with Co ions

Appl. Phys. Lett. 93, 232504 (2008); 10.1063/1.3040696

$\mathrm{Zn}$ and $\mathrm{ZnO}$ nanoparticles fabricated by ion implantation combined with thermal oxidation, and the defect-free luminescence

Appl. Phys. Lett. 88, 153119 (2006); 10.1063/1.2193327

p type doping of zinc oxide by arsenic ion implantation

Appl. Phys. Lett. 87, 192103 (2005); 10.1063/1.2128064

Space-resolved photoluminescence of $\mathrm{ZnS}: \mathrm{Cu}, \mathrm{Al}$ nanocrystals fabricated by sequential ion implantation Appl. Phys. Lett. 84, 2397 (2004); 10.1063/1.1689738

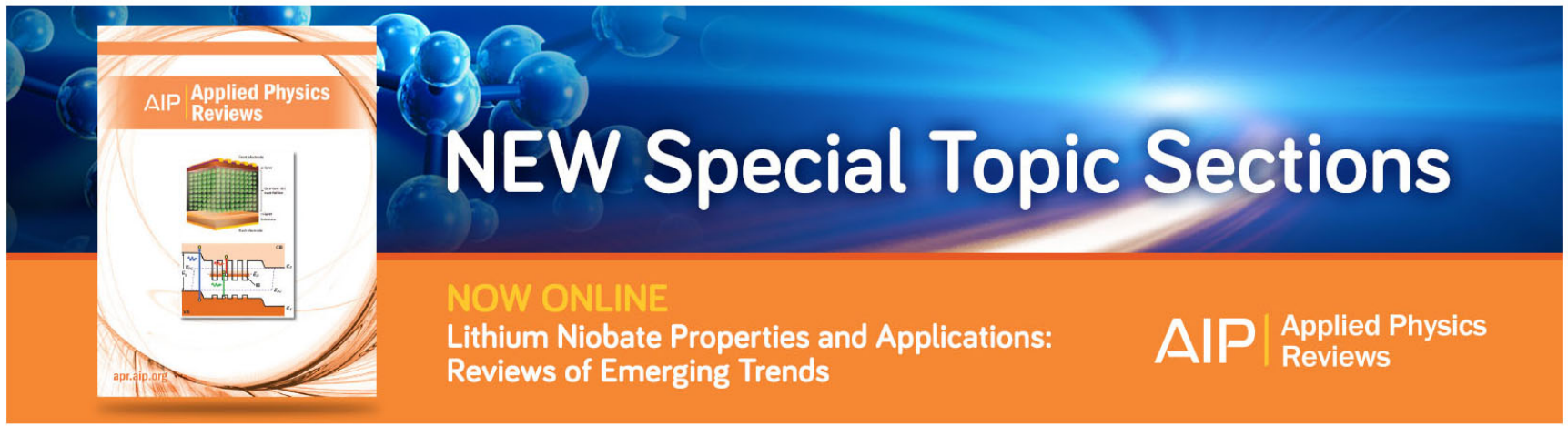




\title{
Substrate lattice relaxations, spectral distortions, and nanoparticle inclusions of ion implanted zinc oxide
}

\author{
Y. Wang, ${ }^{1, \text { a) }}$ B. Ma, ${ }^{1}$ W. Zhang, ${ }^{1}$ D. Li,${ }^{1}$ Y. Zhao, ${ }^{1}$ A. A. Finch, ${ }^{2}$ and P. D. Townsend ${ }^{3}$ \\ ${ }^{1}$ School of Science, China University of Geosciences, Beijing 100083, China \\ ${ }^{2}$ Department of Earth \& Environmental Sciences, University of St Andrews, St Andrews, Fife KY16 9AL, \\ United Kingdom \\ ${ }^{3}$ Physics Building, University of Sussex, Brighton BN1 9QH, United Kingdom
}

(Received 22 June 2015; accepted 18 August 2015; published online 1 September 2015)

\begin{abstract}
Low temperature radioluminescence and thermoluminescence spectra of $\mathrm{ZnO}$ track numerous changes produced by copper ion implantation into the surface layer. A significant, but unexpected, feature is that the bulk crystal becomes modified by the stress generated in the surface layer. This is reflected by the energy of intrinsic band gap emission. There are also differences in the spectra and peak temperatures of the thermoluminescence components, consistent with such a structural relaxation. The copper implant layer is both absorbing and reflective, so this introduces major distortions on the radioluminescence component from the bulk region, since the bulk luminescence signals are transmitted through, or reflected from, the implant layer. The temperature dependence of the spectra includes anomalies that are typical of changes driven by phase transitions of nanoparticle inclusions. Overall, the features of bulk relaxation, spectral distortion, and detection of nanoparticle inclusions are rarely considered for ion implanted luminescence studies, but the data suggest they are almost inevitable in a wide range of implanted materials. (C) 2015 AIP Publishing LLC. [http://dx.doi.org/10.1063/1.4929826]
\end{abstract}

\section{INTRODUCTION}

Zinc oxide is a wide band-gap direct transition semiconductor $(\sim 3.37 \mathrm{eV})$ with the potential to be used in extremely diverse types of devices. The trial, and proposed, uses of $\mathrm{ZnO}$ range from applications producing near-UV and visible light emission, to phosphors, piezoelectric devices and transducers, varistors, and transparent conducting films. ${ }^{1-6} \mathrm{ZnO}$ also exhibits large optical nonlinearities that can be exploited in optical devices. Many of the device applications are therefore being explored with ion implantation routes to modify and control the near surface properties. There are numerous articles concerned with changes controlled by ion implantation in $\mathrm{ZnO}$ with ions ranging from $\mathrm{H}, \mathrm{N}, \mathrm{Si}, \mathrm{Ar}, \mathrm{Ti}, \mathrm{Mn}, \mathrm{Ga}$, $\mathrm{Cu}, \mathrm{As}, \mathrm{In}, \mathrm{Er}, \mathrm{Tb}, \mathrm{Au}$, etc. ${ }^{7,8}$ At least 20 of these studies have used luminescence to monitor the property changes.

Luminescence is valuable as it is remarkably sensitive, and so responds not only to the impurities and defects created by implantation but also to thermal treatments that modify intrinsic defect sites, and their association with the implants. In addition to the normal luminescence technique, recently the confocal micro-photoluminescence has been widely applied to detect the ion beam modified lattice changes. ${ }^{9-11}$ Indeed, the use of luminescence analysis for implanted materials is widespread, both for semiconductors and insulators. One assumes that the emission spectra offer an accurate indication of the changes induced in the surface implanted layer. Additionally, there is the tacit assumption that the underlying material is not significantly modified. The data presented here challenge both assumptions.

\footnotetext{
${ }^{\text {a) }}$ Author to whom correspondence should be addressed. Electronic mail: wyfemail@gmail.com
}

The first feature, of distorted spectra, is not unexpected as in the case of implants that produce optical absorption (such as copper implants). The luminescence generated includes signals from the implant layer and the underlying material, but both are strongly modified by the absorption within the implanted zone. The distortions in signal are particularly evident if the excitation penetrates to a greater depth than the implant. Whilst this is invariably true to some extent, the present results obtained by radioluminescence (RL) are guaranteed to stimulate both the implant and the underlying bulk substrate. $\mathrm{RL}$ is therefore particularly valuable as a method of emphasising that spectral distortions occur. It is not just a problem of RL but equally present in cathodoluminescence and optically excited luminescence studies. However, with the shallower excitation depths, it is difficult to demonstrate, and so the recorded spectra are invariably assumed to be the true signals. Therefore, spectral distortions of the luminescence analysis caused by optical absorption are widespread, but unfortunately often overlooked.

The second feature, of changes in the thick substrate beneath the implanted region, is certainly evident in the data presented here for $\mathrm{ZnO}$ and considered in earlier $\mathrm{Cu}$ and $\mathrm{Tb}$ implant studies of $\mathrm{ZnO} .{ }^{12-14}$ There is, therefore, the potential that similar bulk changes from surface implants may be a more general feature, but they have rarely been discussed or sought, but clearly should be.

Whilst luminescence data are recognised as being valuable in identification of impurities that modify the emission spectra (e.g., rare earth impurities have unique line emission signals), the power of the technique to respond to the presence of nanoparticle inclusions, or phase transitions of either the host or the inclusions, is generally ignored. Nevertheless, 
in the present data for the $\mathrm{ZnO}$, both features are reported. The critical measurements that reveal nanoparticle inclusions of impurities, such as trapped water, or gases of oxygen, nitrogen, or carbon dioxide, require temperature dependent spectral data (employed here). If the nanoparticle inclusions undergo phase transitions, there are changes in both pressure and electronic interactions, not just at their immediate interfaces, but in ways that modify the entire sample. Hence, very small impurity concentrations, in the form of inclusions, can influence and, in some cases, dominate the bulk luminescence conditions. In the present article, examples are demonstrated with $\mathrm{ZnO}$. The technique has been reported and reviewed, ${ }^{15}$ with many other materials. Unfortunately, the literature of such effects is rather limited, primarily because the method has not been applied due to equipment limitations of most luminescence systems. Indeed, most examples so far are based on experiments with the same system. ${ }^{16}$

\section{EXPERIMENTAL DETAILS}

Nominally pure $\mathrm{ZnO}$ samples were used in this work for the RL measurements, and the samples are from Hefeikejing Materials Technology Co., Ltd., China. The sample size of the $\mathrm{ZnO}$ is $10 \times 10 \times 0.5 \mathrm{~mm}^{3}$ with the direction of $\langle 0001\rangle$. One side of the sample has been polished. The implantations with copper ions were to a fluence of $2 \times 10^{16} / \mathrm{cm}^{2}$ and the energy is $50 \mathrm{keV}$.

RL signals excited by X-rays were collected by a high sensitivity luminescence system at the University of $\mathrm{St}$ Andrews. The optics of this spectrometer had been reported
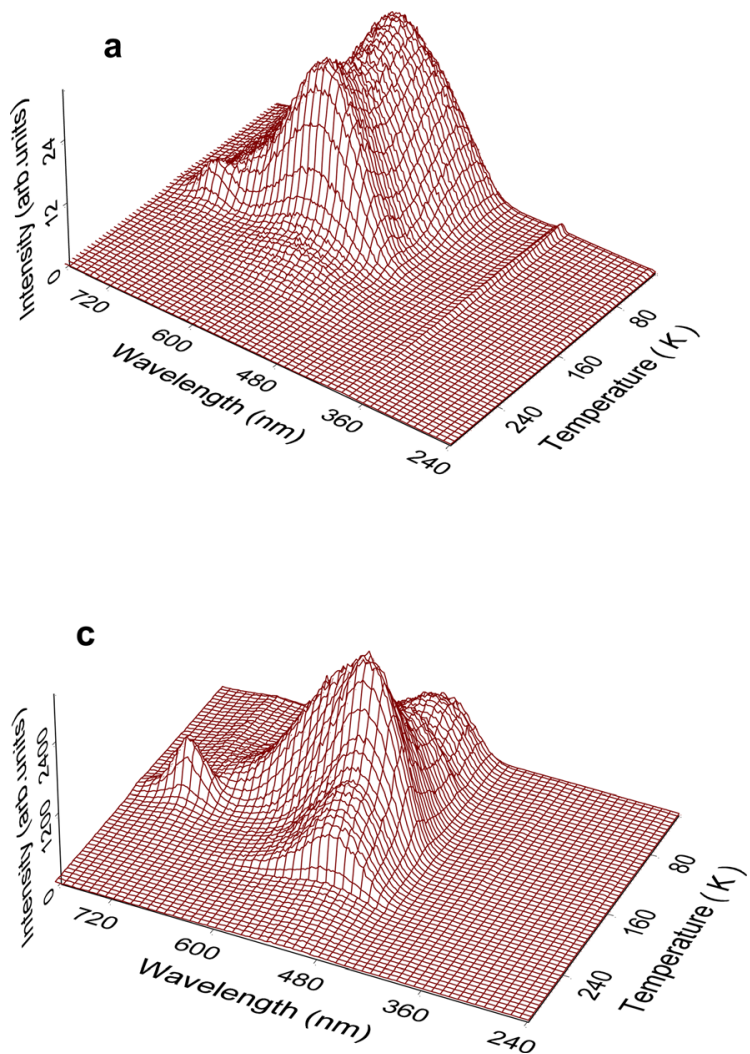

previously, ${ }^{16}$ but significant improvements in detector efficiency and time resolution are now available. The wavelength dispersed spectra are corrected for the response of the overall spectrometer system.

A low temperature stage was operated from 20 to $300 \mathrm{~K}$ and a high temperature stage extended the range to $400{ }^{\circ} \mathrm{C}$ (i.e., 20 to $673 \mathrm{~K}$ overall). A heating rate of $15 \mathrm{~K} / \mathrm{min}$ was used for high temperatures, but a lower rate of $6 \mathrm{~K} / \mathrm{min}$ was required to avoid temperature gradients in the sample at low temperatures (i.e., the thermal conductivity is poor within this range).

The RL was excited with an X-ray energy of $15 \mathrm{keV}$ with radiation dose rates of $\sim 1$ Gy per minute at low temperature, or $\sim 10$ Gy per minute at high temperature.

Both RL and low temperature thermoluminescence (TL) data are reported.

\section{RESULTS AND COMMENTS}

Radioluminescence data are shown in Fig. 1 in the form of isometric plots for original and copper implanted samples, together with implanted specimens that had been annealed at $1000^{\circ} \mathrm{C}$ and either slowly or quickly cooled to room temperature. At first sight, there are similarities between the four data sets in terms of the overall broad band emission patterns and their temperature dependencies. The lower energy signals in the 450 to $800 \mathrm{~nm}$ range predominately relate to defects and sites in the $\mathrm{ZnO}$ host. However, reference to the contour maps of Fig. 2 reveals that not only the positions of the wavelength maxima are not identical, but also there are
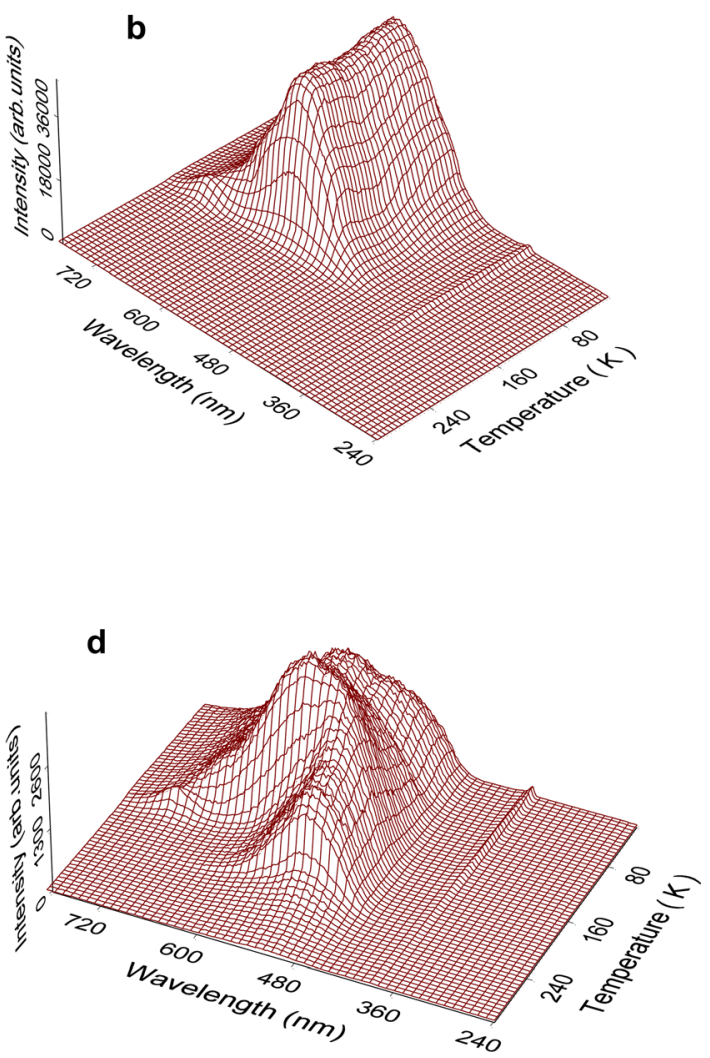

FIG. 1. Isometric plots of $\mathrm{ZnO}$ radioluminescence spectra below room temperature; (a) is an original sample, pure, (b) is for a copper implanted sample, C, (c) copper implanted and annealed with slow cooling, and (d) is with fast cooling. 

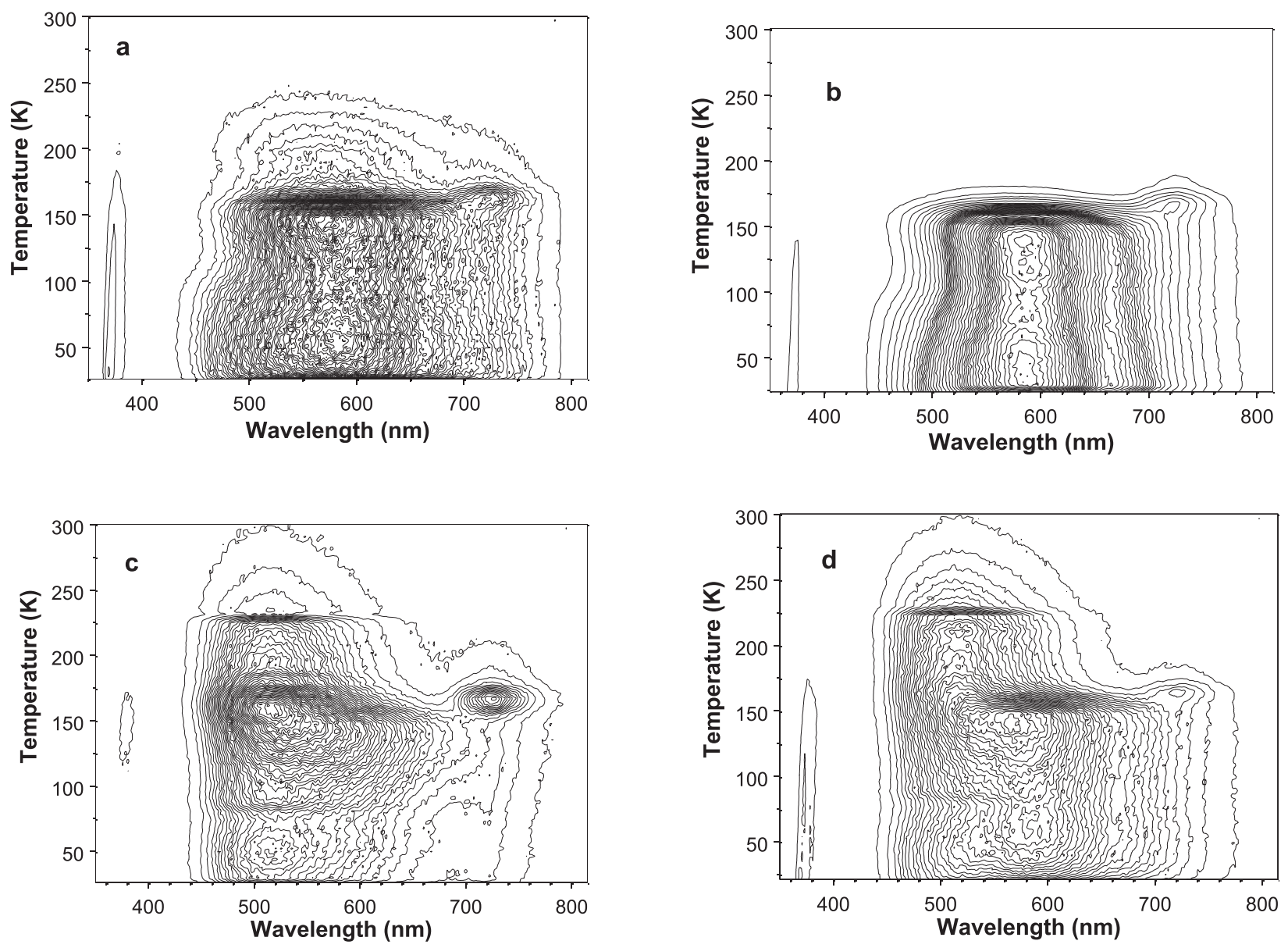

FIG. 2. Contour plots of $\mathrm{ZnO}$ radioluminescence spectra below room temperature; (a) is an original sample, pure, (b) is copper implanted sample; (c) copper implanted and annealed with slow cooling, CAS, and (d) with fast cooling, CA

different mixtures of component peaks. Finally, one sees there are shifts in the peak temperatures of the RL signals. The changes are partly expected as the RL signals were collected for emission exiting the crystal from the implant face. Since the X-rays excite the entire sample, this means the emission is dominated by RL from the substrate, which is nearly one thousand times thicker than the input layer. However, the copper implant introduces strong optical absorption so the measured total RL is from the bulk, but distorted by the implant absorption. The annealing treatments not only reduce the absorption from the implant but also cause changes in the intrinsic defect structures. Hence, there are yet further differences between the slow and fast cooled samples.

Figures 1 and 2 show narrow line emission features at wavelengths less than $400 \mathrm{~nm}$. These peaks correspond to intrinsic band to band transitions and related exciton states. The emission reveals more than one component at the lower temperatures, and Figure 3 presents the observed pattern at $26 \mathrm{~K}$ for an unimplanted sample. Deconvolution into three peaks is possible in terms of Gaussian shaped bands peaked at $3.12,3.19$, and $3.25 \mathrm{eV}$. Note that this data set of signals was obtained in a wavelength dispersed domain with a fixed bandwidth $(\mathrm{d} \lambda)$ (and typically viewed in terms of $I(\lambda) d \lambda$ versus $\lambda$ ). For analysis, they have been transformed into the physically more meaningful energy domain $(I(E) d E$ versus $E)$. No attempt has been made to perform the transformations and deconvolution for the longer wavelength emission bands of the implanted samples, since it is not possible to offer adequate correction factors to the collected data to offset distortions by absorption of light in the implant zone.

Figures 1 and 2 offer a broad pattern of changes, but the differences are better resolved in comparisons of spectra produced at different temperatures. Examples are shown in Fig. 4 that contrast signals at 20,135, and $170 \mathrm{~K}$. Whilst the absorption/reflection properties of the copper implant layer

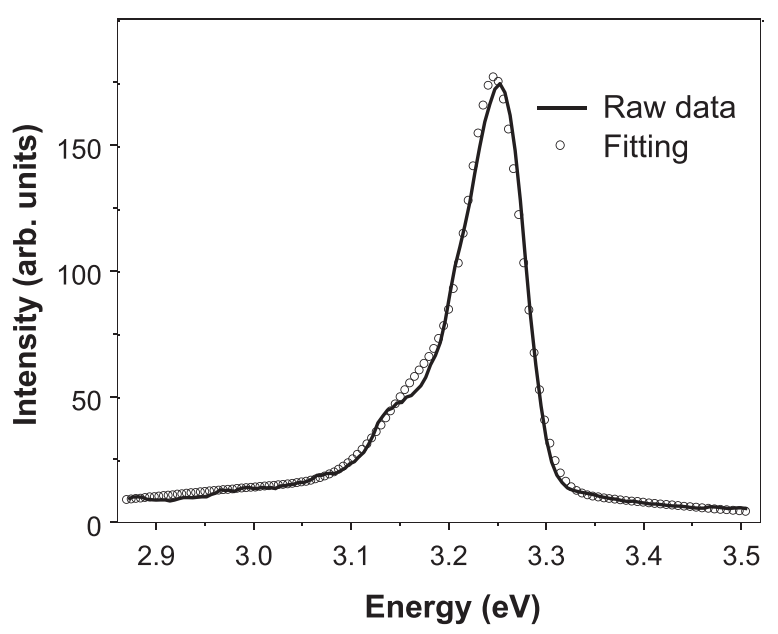

FIG. 3. Energy plot of the RL emission from $\mathrm{ZnO}$ at $26 \mathrm{~K}$. 

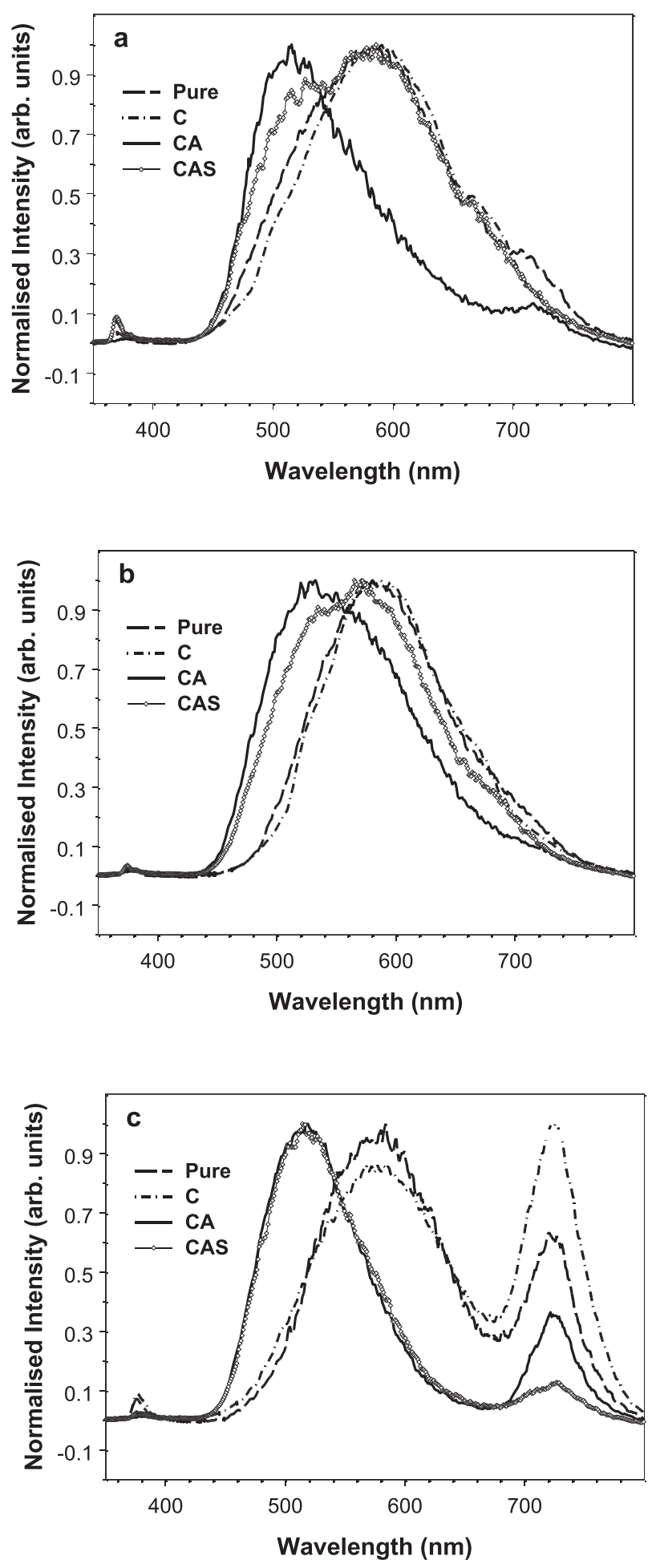

FIG. 4. Comparison of RL spectra for the samples at (a) $30 \mathrm{~K}$, (b) $135 \mathrm{~K}$, and (c) $170 \mathrm{~K}$

are modifying the signal transmitted of the RL from below the shallow implant layer, it is nevertheless clear that the heat treatments are making significant changes. For example, annealing has reactivated signals above $\sim 170 \mathrm{~K}$ and also introduced a rapidly changing intensity step near $225 \mathrm{~K}$. The contour maps include some high density contour features (i.e., regions of steeply changing intensity) at the same fixed temperatures that in earlier materials were indicative of phase transitions of inclusions. These will be discussed later.
The data reveal that in all cases the small peak that is centered near $725 \mathrm{~nm}$ is not at a constant temperature, and it is normally at slightly higher temperatures than the emission maxima at shorter wavelengths. This temperature difference was precisely the same observation for the TL emission reported earlier. ${ }^{14}$ It is therefore useful to contrast the TL signals from the pure and copper doped samples, both before and after annealing. The data are shown in Fig. 5 in the form of isometric plots and contour maps.

Annealing has activated additional low temperature glow peaks and changed the apparent relative intensities of the green to red signals. Spectral intensity data are clearly suspected if there is absorption of the luminescence as it is transmitted through the implant layer, but since the TL is primarily from the $99.9 \%$ of the crystal that is not implanted, the changes in peak temperatures are clear evidence that the defect structures are modified by the stress generated by the copper implant layer, and also by the rate at which annealed samples were cooled (i.e., these change the contributions of intrinsic defects and their association with impurities etcetera). Hence, evidence that there have been structural variations in the bulk unimplanted volume is uniquely demonstrated by a comparison of the peak temperatures, and the shapes, of the main green and red TL signals. Examples are shown in Fig. 6 for the TL in selected wavelength regions.

Numerically, the changes are detailed in Table I, which lists the wavelengths where peaks are observed in the thermoluminescence and their temperatures. The main point to note is that the peak temperatures shift between the four samples. This is of particular significance as it is demonstrating that stress from the implants, as well as heat treatments, is modifying the bulk material. So, the implant has made a significant perturbation of the entire host lattice (i.e., to distances some 1000 times further than the implant). Note that the variations in wavelength for the green peak are difficult to quantify as they reflect the problems of absorption that distort the spectra in the case of the implanted samples.

The copper implant has shifted the peak temperatures by between $25^{\circ}$ and $13^{\circ}$, but after annealing the value for the red peak is similar to that of the original unimplanted sample. A second anomalous feature is that the higher temperature variants tend to be narrower than the low temperature glow peaks (this is contrary to normal TL behaviour), and it suggests that there is an unusually rapid decay in luminescence efficiency with increasing temperature towards $170 \mathrm{~K}$.

A final confirmation that the spectra recorded through the implant layer are being distorted by the layer can be seen in Fig. 7 in which the RL signal has been recorded with the implant face at the bottom of the sample (i.e., the RL light travels directly to the spectrometer). The data of Fig. 7 should be compared with those in Figs. 1(a) and 2(a), as in each case the primary signal is from unimplanted $\mathrm{ZnO}$. The two data sets are not identical. In part, this will be because moving the implant to the underside during recording is not a perfectly unperturbed situation as the implant layer will act as a wavelength selective reflective mirror that contributes to the overall signal. Nevertheless, it is clear that in the green region the peak wavelength is much closer to that of the original unimplanted sample than data collected with the copper 

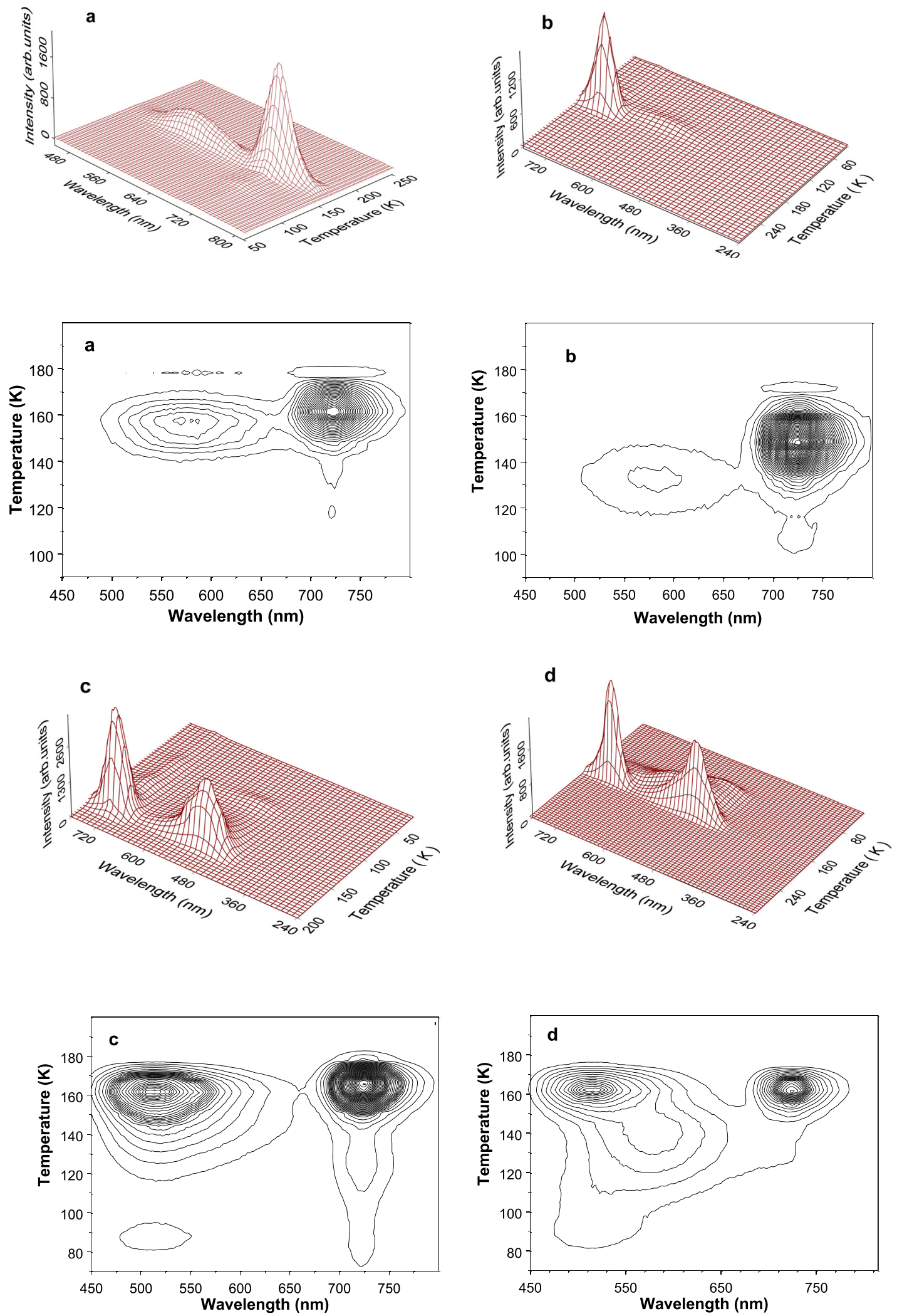

FIG. 5. Comparisons of thermoluminescence for (a) an original sample, pure, (b) copper implanted sample; (c) copper implanted and annealed with slow cooling, and (d) with fast cooling.

implant layer uppermost. Nevertheless, there are differences in relative intensities of the peaks, and, for example, at $20 \mathrm{~K}$, the initial signal includes a feature that is displaced towards the blue end of the spectrum. The contour plot indicates a number of horizontal dark zones where there are rapid changes in intensity with temperature. The most dramatic of these is seen in Fig. 8, which indicates that for the broad bands there is an intensity notch near $\sim 54 \mathrm{~K}$. The anomaly does not appear in the band to band transitions near $400 \mathrm{~nm}$, nor it is an artefact of detector saturation since it is not at the 

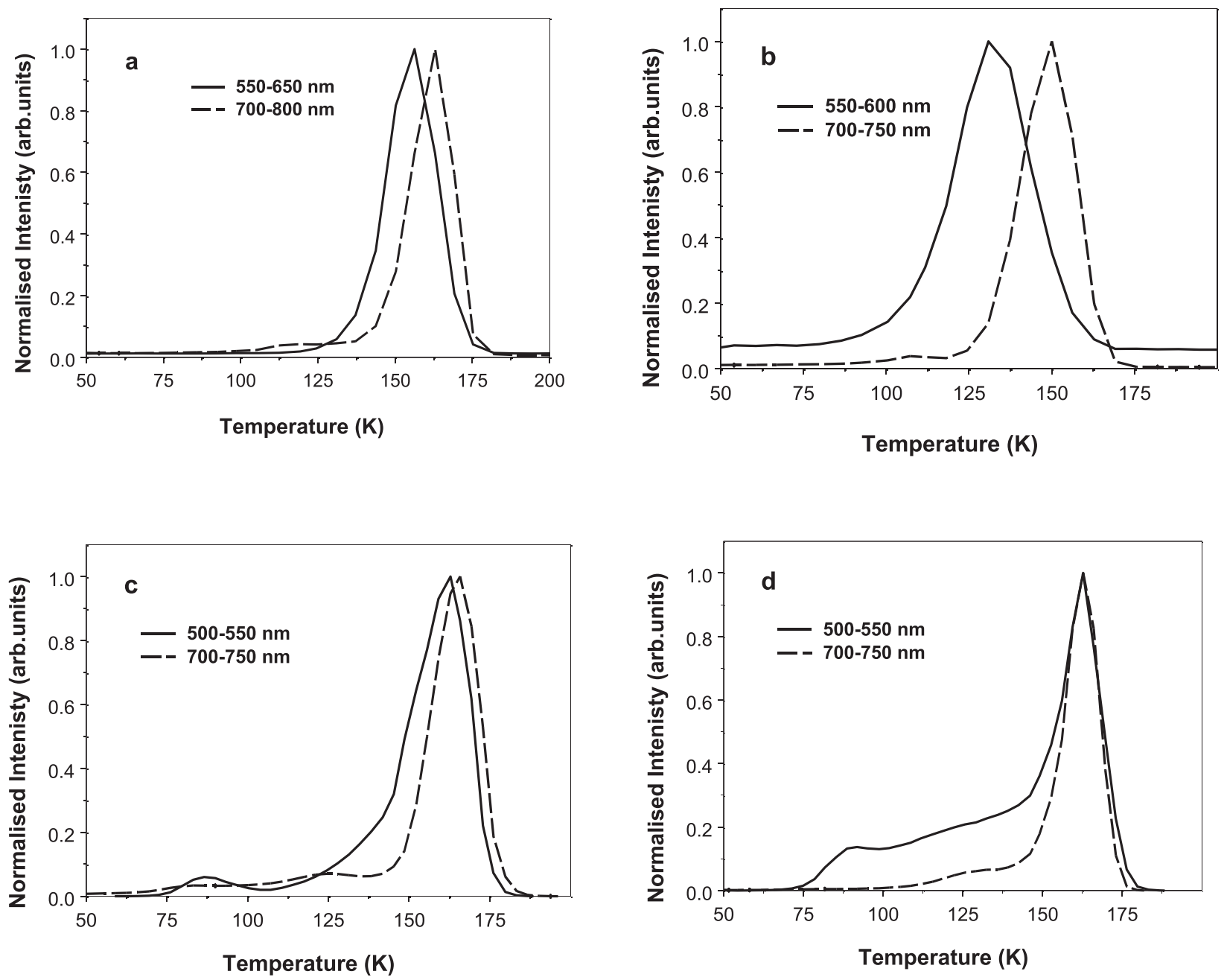

FIG. 6. TL slice for the samples (a) is pure $\mathrm{ZnO}$, (b) is copper implanted, (c) implanted and annealed with a slow cooling, and (d) for the fast cooling after annealing.

region of greatest intensity. The later discussion will suggest it is linked to a phase transition of nanoparticle inclusions.

Finally, the intrinsic properties of the lattice structure are addressed via the short wavelength emission data from the band to band signals. For an unmodified substrate, these should be identical in Figs. 1(a) and 7. They are not. The comparison is made in Fig. 9, which shows that in detail there are significant spectral shifts between the two data sets at low temperature, but they tend towards the same values at the highest temperatures. This will be discussed as unequivocal evidence for a change in the lattice structure of the bulk of the implanted sample.

TABLE I. The TL peak position for the samples.

\begin{tabular}{|c|c|c|c|c|c|c|}
\hline \multirow{2}{*}{$\begin{array}{l}\text { Sample } \\
\text { Unimplanted }\end{array}$} & \multirow{2}{*}{$\begin{array}{l}\text { Label } \\
\text { Pure }\end{array}$} & \multicolumn{2}{|c|}{$\begin{array}{c}\text { Peak } \\
\text { positions } \\
(\mathrm{nm})\end{array}$} & \multicolumn{2}{|c|}{$\begin{array}{c}\text { Peak } \\
\text { temperatures } \\
(\mathrm{K})\end{array}$} & \multirow{2}{*}{$\begin{array}{c}\begin{array}{c}\text { Difference } \\
(\mathrm{K})\end{array} \\
7\end{array}$} \\
\hline & & 575 & 723 & 156 & 163 & \\
\hline $\mathrm{Cu}$ implanted & $\mathrm{C}$ & Broad & 724 & 131 & 150 & 19 \\
\hline $\begin{array}{l}\text { Anneal and } \\
\text { slow cool }\end{array}$ & CAS & 518 & 724 & 163 & 166 & 3 \\
\hline $\begin{array}{l}\text { Anneal and } \\
\text { fast cool }\end{array}$ & $\mathrm{CA}$ & 534 & 724 & 163 & 163 & 0 \\
\hline
\end{tabular}

\section{DISCUSSION}

\section{A. Structural relaxations of substrates}

One objective of this study was to confirm the proposition that high fluence implants in $\mathrm{ZnO}$ results in a structural change of the entire block of the substrate. Earlier $\mathrm{ZnO}$ data had revealed that both $\mathrm{Cu}$ and $\mathrm{Tb}$ implants displaced the TL emission patterns from that of the unimplanted material. ${ }^{12-14}$ This type of change was observed again in the current study, as shown by Fig. 5. Since the TL is primarily from the nonimplanted volume, then a change in TL peak temperatures is consistent with a distortion of the original lattice structure. Additionally, from the present data, the alterations produced in the luminescence from band to band transitions are also consistent with a change in the band gap at low temperature. Such a change is expected if there is a structural relaxation of the wurtzite lattice driven by the stress field created in the surface implant region. Since the $\mathrm{ZnO}$ is used in piezooptics, there is certainly the potential for such a change.

For the unimplanted material, the temperature dependence of the band gap (Fig. 3) is well described by temperature induced lattice dilatation and electron-lattice interaction as described by the model of Varshni. ${ }^{17}$ The data shown in Fig. 9, for the unimplanted $\mathrm{ZnO}$, fit a Varshni type formula 

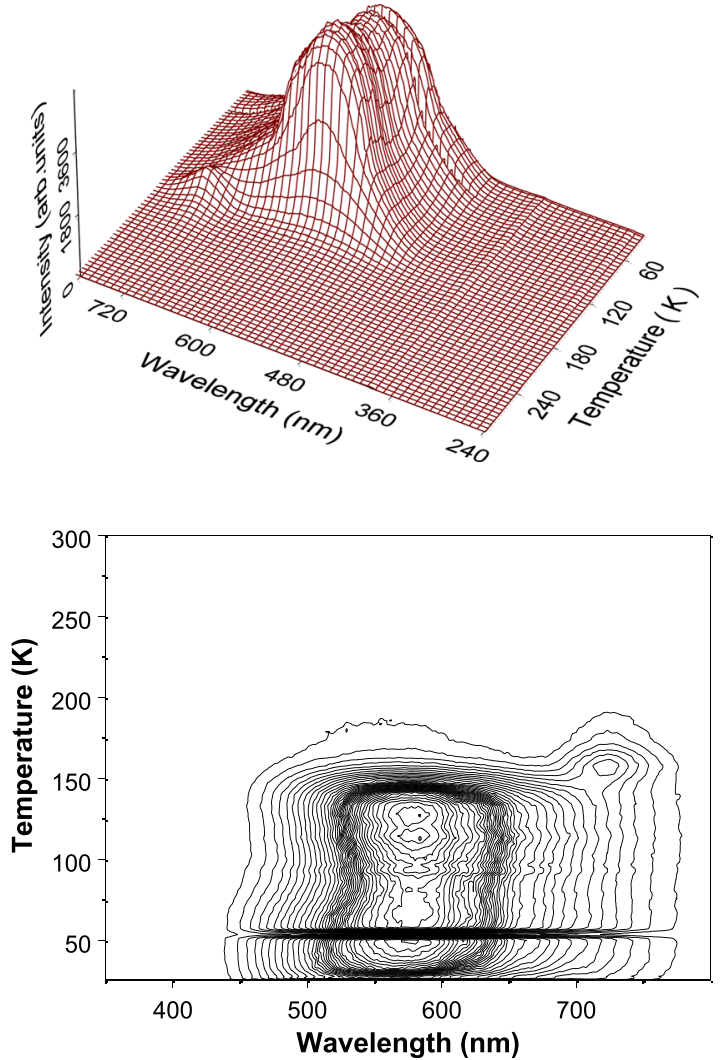

FIG. 7. Radioluminescnce measured with the implant face downwards.

over the entire range from 20 to $673 \mathrm{~K}$ via the equation of the form

$$
E_{g}=E_{0}-\alpha T^{2} /(T+\beta),
$$

where $E_{g}$ is the band gap at an absolute temperature $T, E_{0}$ is the band gap at zero, $\alpha$ and $\beta$ are the Varshni thermal coefficients related with given materials. For the fitting parameter

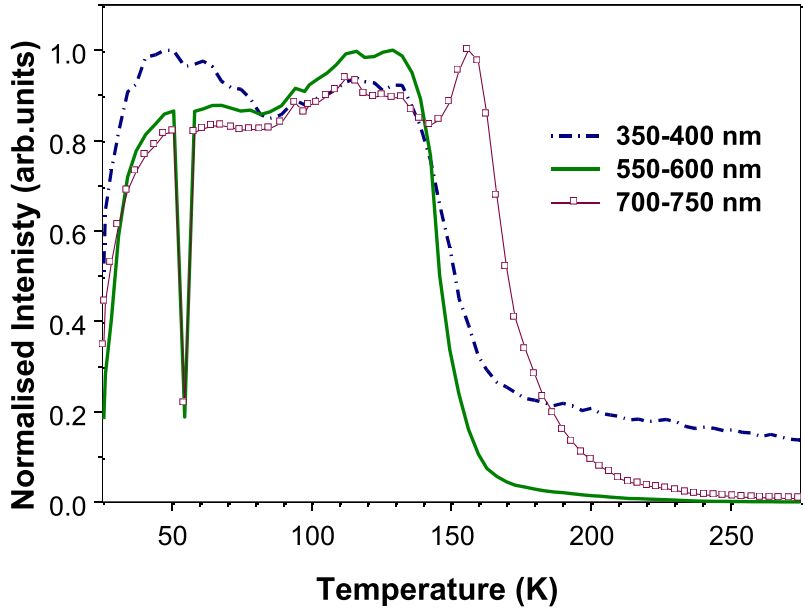

FIG. 8. Sections taken from the isometric plot of Figure 7. Note the intensity dip near $54 \mathrm{~K}$ for the long wavelength emission.

needed here, it implies the band gap at $0 \mathrm{~K}$ is $3.249 \mathrm{eV}$, the other two parameters are $9.147 \times 10^{-4} \mathrm{eV} / \mathrm{K}$ and $256.41 \mathrm{~K}$.

For the implanted $\mathrm{ZnO}$, Figure 9 shows displacement of the low temperature curve, which would match a structural relaxation. The perturbed values describe a slightly more complex temperature pattern, which may imply the creation of some phase instabilities in the stressed material, and fitting gives a reduced band gap luminescence at $0 \mathrm{~K}$, caused by the $\mathrm{Cu}$ implant, as $3.226 \mathrm{eV}$.

Since the proposition that a surface implant can modify the entire crystal is so rarely considered, it is essential to offer other examples of evidence that support this suggestion of long range stress effects. There are many examples of optical waveguides formed by ion implantation, where the damage from the implant has amorphized a crystalline lattice in a narrow zone below the guide region and reduced the refractive index so as to form an optical barrier. ${ }^{18-20}$ Waveguide lasers based on rare earth ions then have
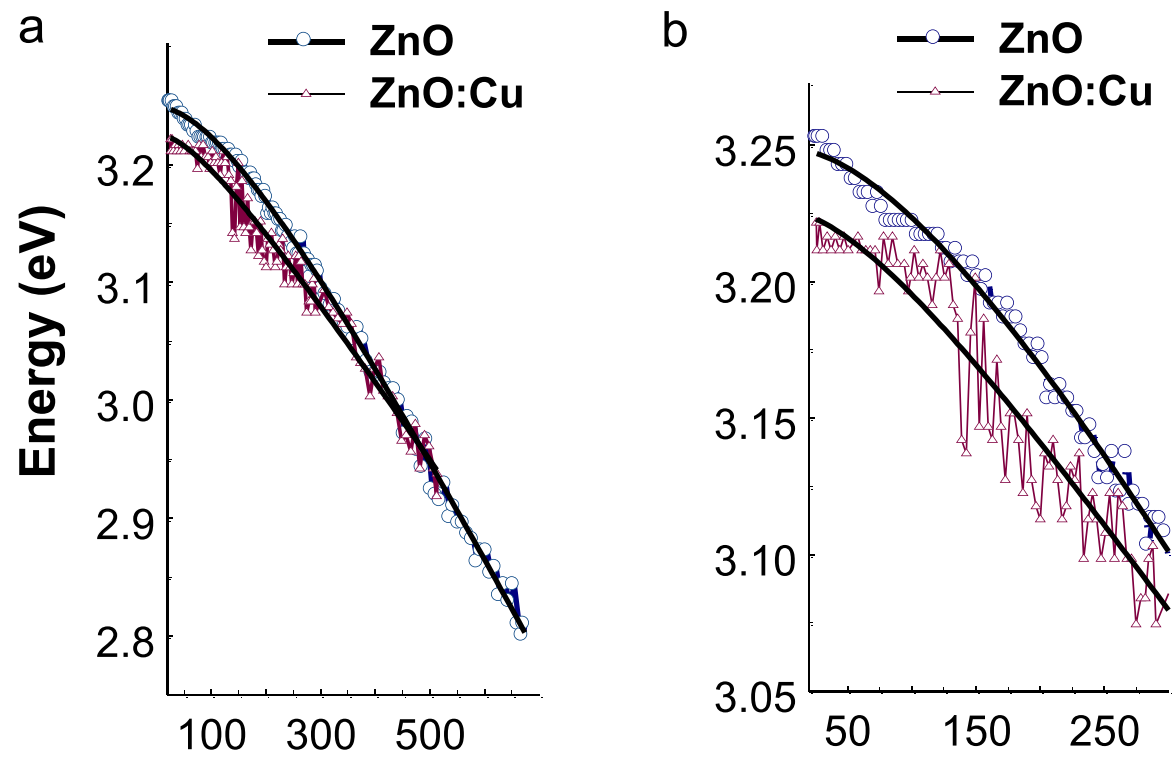

FIG. 9. Low temperature RL for $\mathrm{ZnO}$ and a $\mathrm{ZnO}: \mathrm{Cu}$ sample monitored with the implant face down (a) is a comparison over the entire temperature range and (b) emphasises the differences observed at low temperature. The fitting energies are $3.226 \mathrm{eV}$ for $\mathrm{ZnO}: \mathrm{Cu}$ and $3.248 \mathrm{eV}$ for pure $\mathrm{ZnO}$. 
emission spectra that are altered both in wavelength and relative transition line efficiency. The examples are clear and indicate stress effects exist over waveguide of several micrometers width (e.g., at least $8 \mu \mathrm{m}^{18}$ ) in many materials. ${ }^{18,19,21-23}$

Far longer range stress is evidenced in studies of luminescence from strontium titanate (STO), where the radioluminescence has demonstrated phase changes initiated by the stress. STO is unusual in that below room temperature, there are a number of crystalline phase changes and relaxations that are known to occur. These are extremely sensitive to impurities, intrinsic defects, and stress. Implantation into a STO surface generates a strong stress field across the entire crystal, which then induces the appearance of these metastable phases. For STO, the changes were very obvious as each phase has quite different and characteristic emission spectra. With STO, the various phases were seen only in the heavily implanted (and therefore stressed) crystals. ${ }^{15,24}$ Indeed, the more general possibility of monitoring phase transitions by noting the discontinuities in emission spectra is well documented for a large number of insulator and other materials, as noted in various reviews. ${ }^{15}$

The waveguide and STO examples support the model for bulk relaxation of $\mathrm{ZnO}$, driven by stresses related to high dose surface implantation. Relaxation in $\mathrm{ZnO}$ is not unexpected as it is already used as an electro-optic material.

\section{B. Heat treatments and changes in intrinsic defects and structure}

The thermoluminescence data for the $\mathrm{ZnO}$ experiments at low temperature have two main peaks at slightly different temperatures for different emission bands. These are seen in pure, as well as copper or terbium implanted samples. Models for the various TL sites therefore do not primarily require impurity sites but suggest that they are intrinsic sites that are readily associated with impurities and/or other intrinsic defects. In the stress conditions of the copper implants, there are significant differences in the glow peak temperatures compared with unimplanted samples. Similarly, the examples of annealing at $1000{ }^{\circ} \mathrm{C}$ followed by either slow or rapid cooling modify many intrinsic defect properties (such as oxygen vacancy concentrations and their charge states); additionally, the high temperature can dissolve clusters of defects (or impurity-intrinsic complexes). Therefore, the rate of cooling influences the concentrations of associated defects retained at room temperature. Such processes are evident in the present TL data. Not only are the TL signals moving in terms of their peak temperatures but also in terms of the number of minor features.

The emission spectra of original and unimplanted samples have peaks near 725 and $575 \mathrm{~nm}$, but after the heat treatment anneals, the shorter wavelength feature moves across to near 518 to $534 \mathrm{~nm}$, although some minor evidence of emission near $570 \mathrm{~nm}$ also exists (Fig. 5(d)). Overall, this implies the stress and heat treatments move the stability of the original charge trapping sites (i.e., the peak temperatures move) but the thermal treatments modify the recombination sites (i.e., the "green" emission wavelengths are altered).
Overall, this implies a lively interplay of defect sites, and therefore no specific model will be offered. Note that earlier models for $\mathrm{ZnO}$ luminescence emission ${ }^{25-34}$ were quite speculative and in hindsight the suggestion that the green signals resulted from copper impurities seems unlikely, as the data for $\mathrm{Cu}$ and $\mathrm{Tb}$ implants are very similar.

\section{Role of the implanted layer on spectral distortions}

Analysis of implanted layers by luminescence is a routine technique for both semiconductor and insulator materials, and the tacit assumption is that the signals are reliable and not significantly distorted by absorption within the implant zone. Pragmatically, they can still offer comparative information even when spectral distortions occur. For excitations that only penetrate over the same depth scale as the implant, the contributions from the substrate are less important than in the current radioluminescence study. Whilst it is an extreme situation, it strongly emphasises how absorption and reflectivity within the implant layer can totally distort the recorded spectra. An additional consideration is that, when stress fields are involved, there is the further possibility or producing polarised emission, and this will influence the recorded spectra since the diffraction gratings of the spectrometer are strongly polarization dependent as a function of wavelength.

For the copper implanted $\mathrm{ZnO}$, there will be oxygen loss, or movement away from the implant zone, and excess metal ( $\mathrm{Cu}$ and/or $\mathrm{Zn}$ ) will form a range of depth and concentration dependent nanoparticles. In the case of copper particles, there is a strong plasmon resonance near $620 \mathrm{~nm}$.

Examples of copper implants suggest the nanoparticle size is around 2 to $3 \mathrm{~nm}$, but the sizes vary with concentration and thermal processing. ${ }^{35,36}$ However, detailed analyses of silver implants suggest a wide range in sizes will exist, and around $75 \%$ of the implant is in nanoparticles of less than $3 \mathrm{~nm}$ diameter. ${ }^{37}$

The shape of this absorption band will vary slightly with particle size. Consequently, the reflectivity from the implant surface and the internal boundaries will differ. For RL, where most of the luminescence is generated in the bulk of the sample, the absorption will dramatically alter the emission spectrum of light that is transmitted through the implant layer. For the alternative geometry of implant side away from the spectrometer, the layer will still not be neutral as the interface "mirror" is wavelength dependent.

Such distortions are apparent in the current RL data, and to indicate the pattern they impose on the spectra, Figure 10 shows the transmission and reflectance for a copper ion implanted glass sample. This has the expected maximum absorption near the plasmon resonance region around $620 \mathrm{~nm}$, together with reflectivity features across the entire spectral range being monitored. The variations in different particle sizes across the implant zone are evident via a surface dependent difference in the reflectivity spectrum. In the glass example, the peak transmission near $620 \mathrm{~nm}$ falls to a mere $25 \%$, and reflectance values from the rear face are as high as $\sim 8 \%$. Note because the particle sizes vary with depth, the front and rear face reflectivity values and 

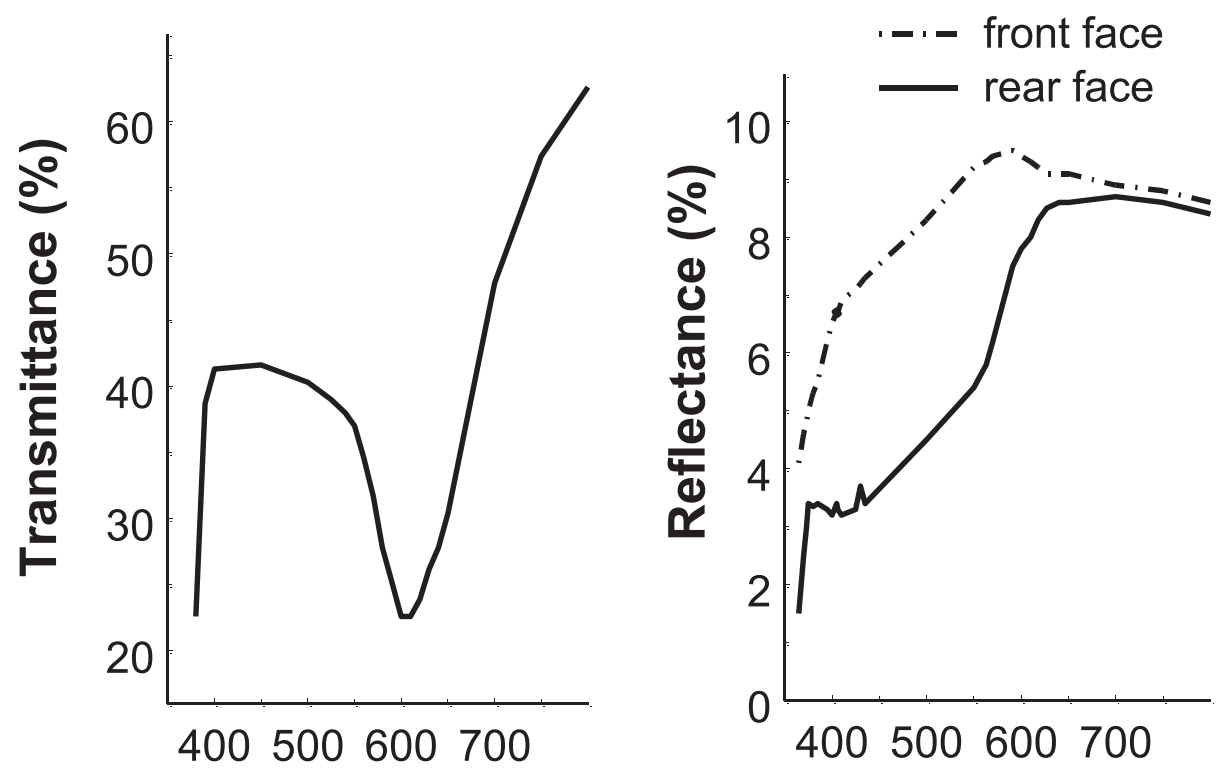

FIG. 10. Examples of the absorption and reflectivity changes produced by the formation of copper implanted nanoparticles. Note the nanoparticle sizes vary with depth, so the front and rear face reflectivities differ. The example here is for $\mathrm{Cu}$ in glass.

\section{Wavelength (nm)}

wavelength dependence are different. In luminescence studies, such features are significant and difficult to correct for. They also have been overlooked in many literature examples.

\section{The role of nanoparticle inclusions}

Many of the impurities that are introduced during the crystal growth phase become bonded into the materials, not only as isolated impurity sites but also as nanoparticle inclusions. Their size may be small, a few tens or more of impurities, but their influence on the lattice luminescence will be far more extensive as the stress fields they generate can influence optical transitions that occur at more than a 100 nearest neighbor sites away from the inclusion. Therefore, impurity concentrations of say, 10 parts per million may influence the entire sample. The equipment used here has been able to sense such events for a wide range of materials, and a very common indicator of nanoparticle inclusions has been apparent when they undergo a low temperature phase transition. The effects are clear since the phase change provides a discontinuity in the pressure conditions. In many examples, there are wavelength displacements of the spectra, and or major discontinuities in intensity, as reviewed, ${ }^{15,38}$ for $\mathrm{MgO}^{39}$ or for Nd:YAG where both spectral and lattice parameter changes were reported from $\mathrm{CO}_{2}$ sublimation within nanoparticles, and ice effects were seen. ${ }^{40}$ Such features are quite obvious from spectral changes of the host lattice that occur when the host undergoes a phase transition.

Particularly repeatable and frequent examples exist for ice inclusions as there is an ice phase change from hexagonal to cubic structures at $\sim 170 \mathrm{~K}$ and a low pressure ice to vapour change near $225 \mathrm{~K}$. Other dramatic events have been noted from inclusion of $\mathrm{CO}_{2}$, as at $\sim 195 \mathrm{~K}$, the material sublimes from solid to gas with a consequent thousand fold increase in pressure. Many of the common gases from air
$\left(\mathrm{O}_{2}, \mathrm{~N}_{2}\right.$, Ar, etc) have caused discontinuities in intensity when there are gas/liquid or liquid/solid transitions, as seen with low grade $\mathrm{MgO}$ crystals. ${ }^{39}$ In some cases, abnormalities in the temperature dependent lattice parameter of the host have been matched to the impurity phase events.

Luminescence is consequently an extremely powerful probe of such nanoparticle inclusions, and it is sensible to consider if there is evidence in the current $\mathrm{ZnO}$ data that might display these trends. Indeed, the data show some relevant abnormalities. The intensity plots of the band edge luminescence include a discontinuity near $170 \mathrm{~K}$ (i.e., the hexagonal/cubic ice transition). There is also an intensity plateau region (from $\sim 50$ to $85 \mathrm{~K}$ ) and slightly erratic steps in the transition energy (from $\sim 70$ to $\sim 95 \mathrm{~K}$ ). Overall, this temperature range covers the transitions of the components of air. The phase transitions for oxygen occur at 54.6 and $90 \mathrm{~K}$, whereas those for nitrogen are at 63.2 and $77.2 \mathrm{~K}$. Trapped air in parts per million is clearly a realistic impurity and one that is difficult to accurately quantify in standard analytical analyses.

Figure 8 is therefore particularly interesting, since here the intensity dip is nominally at $54.6 \mathrm{~K}$ corresponding to the solid to liquid oxygen phase change. The presence of oxygen nanoparticles is feasible since the copper implants not only cause surface loss of oxygen but also drive oxygen deeper into the crystal. If this forms nanoparticles, then there may be the source of the event seen here at $\sim 54.6 \mathrm{~K}$. More speculative is that the contour maps have "cliff" type features near 90,170 , and $225 \mathrm{~K}$. The $90 \mathrm{~K}$ feature could match the liquid/gas phase transition of the oxygen nanoparticles. The contour line patterns have features near 170 and $225 \mathrm{~K}$, which similarly may be evidenced for trapped water ice phase transitions.

One caveat is that phase transition temperatures are cited in terms of bulk materials, not nanoparticles. If there are a range of nanoparticle size inclusions, the overall effects 
may be spread over a temperature range. The inclusions discussed here are insulators, so size effect on phase transition temperatures may be modest. By contrast, metallic bonding causes a very significant temperature range for melting that is particle size dependent.

For these $\mathrm{ZnO}$ data, the proposal is tentative, but important since such events do not appear to be generally considered. By contrast, in other insulator materials where luminescence spectra have been recorded over a continuous temperature range, very clear and intense changes have been noted that match remarkably well to water, $\mathrm{CO}_{2}$, and oxygen transitions. The luminescence intensity events seen here may be less dramatic and clear cut than those in say $\mathrm{Nd}: \mathrm{YAG}^{40}$ or $\mathrm{MgO},{ }^{39}$ but they should be seriously considered. Not least, as even the possibility of having impurity inclusions that can be detected by host luminescence is a topic that most experimentalists are unaware of, and consequently will not normally be considered.

\section{CONCLUSIONS}

The data presented here for copper implanted $\mathrm{ZnO}$ offer evidence for serious distortions of the emission spectra because of copper nanoparticles within the implant layer. This has far wider implications than just for this example as such problems imply that we have a distorted view of the true spectra. A second feature that is not often discussed is that the implanted layer can distort the underlying bulk material. In this example, the measurement of the band edge luminescence shows it has occurred.

Thermoluminescence similarly reveals bulk changes driven by stresses generated in the implant zone.

Finally, there is luminescence evidence for the presence of intensity changes driven by nanoparticle inclusions of oxygen and, albeit more tentatively, of water inclusions.

The main conclusion is that these types of feature may be far more general than has previously been suspected, but using a highly sensitive luminescence spectral analysis, it is possible to detect each type of event.

\section{ACKNOWLEDGMENTS}

The authors would like to thank the support of the Fundamental Research Funds for the Central Universities of China, the National Natural Science Foundation of China (No.11205134), and Beijing Higher Education Young Elite Teacher Project (YETP0640).

${ }^{1}$ C. Klingshirm, R. Hauschild, H. Priller, M. Decker, J. Zeller, and Hr. Kalt, Superlattices Microstruct. 38, 209 (2005).

${ }^{2}$ S. J. Pearton, D. P. Norton, K. Ip, and Y. W. Heo, J. Vac. Sci. Technol. 22, $932(2004)$

${ }^{3}$ S. J. Pearton, D. P. Norton, K. Ip, Y. W. Heo, and T. Steiner, Prog. Mater. Sci. 50, 293 (2005)
${ }^{4}$ D. M. Bagnall, Y. F. Chen, Z. Zhu, T. Koyama, S. Shen, and M. Y. Goto, Appl. Phys. Lett. 70, 2230 (1997).

${ }^{5}$ Z. K. Tang, G. K. L. Wong, and P. Yu, Appl. Phys. Lett. 72, 3270 (1998).

${ }^{6}$ H. Cao, J. Y. Wu, H. C. Ong, J. Y. Dai, and R. P. H. Chang, Appl. Phys. Lett. 73, 572 (1998).

${ }^{7}$ P. P. Murmu, R. J. Mendelsberg, J. Kennedy, D. A. Carder, B. J. Ruck, A. Markwitz, R. J. Reeves, P. Malar, and T. Osipowicz, J. Appl. Phys. 110, 033534 (2011).

${ }^{8}$ J. Kennedy, G. V. M. Williams, P. P. Murmu, and B. J. Ruck, Phys. Rev. B 88, 214423 (2013).

${ }^{9}$ D. Jaque and F. Chen, Appl. Phys. Lett. 94, 011109 (2009).

${ }^{10}$ F. Chen, Y. Tan, and D. Jaque, Opt. Lett. 34, 28 (2009).

${ }^{11}$ D. Jaque, F. Chen, and Y. Tan, Appl. Phys. Lett. 92, 161908 (2008).

${ }^{12}$ T. Karali, N. Can, L. Valberg, A. L. Stepanov, P. D. Townsend, Ch. Buchal, R. A. Ganeev, A. I. Ryasnyansky, H. G. Belik, M. L. Jessett, and C. Ong, Physica B 363, 88 (2005).

${ }^{13}$ A. Cetin, R. Kibar, M. Ayvacıklı, N. Can, Ch. Buchal, P. D. Townsend, A. L. Stepanov, T. Karali, and S. Selvi, Nucl. Instrum. Methods Phys. Res., Sect. B 249, 474 (2006).

${ }^{14}$ Y. Wang, B. Yang, N. Can, and P. D. Townsend, J. Appl. Phys. 109, 053508 (2011).

${ }^{15}$ P. D. Townsend, B. Yang, and Y. Wang, Contemp. Phys. 49, 255 (2008).

${ }^{16}$ B. J. Luff and P. D. Townsend, Meas. Sci. Technol. 4, 65 (1993).

${ }^{17}$ Y. P. Varshni, Physica 34, 149 (1967).

${ }^{18}$ P. D. Townsend, P. J. Chandler, and L. Zhang, Optical Effects of Ion Implantation (Cambridge University Press, Cambridge, 1994).

${ }^{19}$ B. Herreros, G. Lifante, F. Cusso, P. D. Townsend, and P. J. Chandler, J. Phys. D 28, 1687 (1995).

${ }^{20}$ F. Chen, X. L. Wang, and K. M. Wang, Opt. Mater. 29, 1523 (2007).

${ }^{21}$ F. Chen, Laser Photonics Rev. 6, 622 (2012).

${ }^{22}$ Y. Ren, N. Dong, F. Chen, and D. Jaque, Opt. Express 19, 5522 (2011).

${ }^{23}$ Y. Ren, N. Dong, Y. Jia, L. Pang, Z. Wang, Q. Lu, and F. Chen, Opt. Lett. 36, 4521 (2011)

${ }^{24}$ B. Yang, P. D. Townsend, and R. Fromknecht, Nucl. Instrum. Methods. Phys. Res., Sect. B 217, 60 (2004).

${ }^{25}$ D. G. Thomas, J. Phys. Chem. Solids 15, 86 (1960).

${ }^{26}$ W. Shan, W. Walukiewicz, J. W. Ager III, K. M. Yu, H. B. Yuan, H. P. Xin, G. Cantwell, and J. J. Song, Appl. Phys. Lett. 86, 191911 (2005).

${ }^{27}$ V. A. Fonoberov and A. A. Balandin, Appl. Phys. Lett. 85, 5971 (2004).

${ }^{28}$ D. Li, Y. H. Leung, A. B. Djurišić, Z. T. Liu, M. H. Xie, S. L. Shi, S. J. Xu, and W. K. Chan, Appl. Phys. Lett. 85, 1601 (2004).

${ }^{29}$ K. Vanheusden, C. H. Seager, W. L. Warren, D. R. Tallant, and J. A. Voigt, Appl. Phys. Lett. 68, 403 (1996).

${ }^{30}$ A. B. Djurišić, W. C. H. Choy, V. A. L. Roy, Y. H. Leung, C. Y. Kwong, K. W. Cheah, T. K. G. Rao, W. K. Chan, H. F. Lui, and C. Surya, Adv. Funct. Mater. 14, 856 (2004).

${ }^{31}$ N. Y. Garces, L. Wang, L. Bai, C. N. Giles, L. E. Halliburton, and G. Cantwell, Appl. Phys. Lett. 81, 622 (2002).

${ }^{32}$ D. C. Reynolds, D. C. Look, B. Jogai, and H. Morkoç, Solid State Commun. 101, 643 (1997).

${ }^{33}$ J. F. Xu, J. R. Zhang, W. P. Ding, W. Yang, Y. W. Du, J. Zuo, C. Y. Xu, Y. H. Zhang, and Z. L. Du, Solid State Commun. 101, 467 (1997).

${ }^{34}$ L. E. Greene, M. Law, J. Goldberger, F. Kim, J. C. Johnson, Y. Zhang, R. J. Saykally, and P. Yang, Angew. Chem. 42, 3031 (2003).

${ }^{35}$ Y. Takeda, C. Lee, V. V. Bandourko, and N. Kishimoto, Mater. Trans. 43, 1057 (2002)

${ }^{36}$ A. L. Stepanov, Tech. Phys. 50, 285 (2005).

${ }^{37}$ L. C. Nistor, J. Van Landuyt, J. Barton, D. E. Hole., N. D. Skelland, and P. D. Townsend, J. Non-Crystall. Solids 162, 217 (1993).

${ }^{38}$ Y. Wang, B. Yang, and P. D. Townsend, Luminescence 28, 253 (2013).

${ }^{39}$ M. Maghrabi, F. Thorne, and P. D. Townsend, Nucl. Instrum. Methods. Phys. Res., Sect. B 191, 181 (2002).

${ }^{40}$ M. Maghrabi, P. D. Townsend, and G. Vazquez, J. Phys.: Condens. Matter 13, 2497 (2001) 